\title{
Single small enhancing CT lesions in Indian patients with epilepsy: clinical, radiological and pathological considerations
}

\author{
Mathew J Chandy, Vedantam Rajshekhar, Siddhartha Ghosh, Shankar Prakash, \\ Thomas Joseph, Jacob Abraham, Sushil M Chandi
}

\begin{abstract}
Thirty consecutive Indian patients with focal or generalised seizures and single, small ( $<10 \mathrm{~mm}$ ), enhancing lesions on CT scans (SSECTL) were studied. Five patients (Group A) were treated with anticonvulsants alone and did not have a biopsy. In ten patients (Group B) a CT guided stereotaxic biopsy of the lesion was carried out and in the remainder (15-Group C) an excision biopsy of the lesion was carried out following CT guided stereotaxic localisation. In all patients in Group B the lesions were reported as "chronic nonspecific inflammation". In seven of 15 patients in Group $C$ the lesions showed a cysticercus with a granuloma and in a further five the pathology was that of a "parasitic granuloma" but the parasite could not be identified. Biopsy did not reveal a tuberculoma or neoplasm in any of the patients. The lesions studied are the same as "disappearing" CT lesions reported in Indian patients, as in 12 of 15 patients in Groups $A$ and $B$, who could be followed up for more than three months, the lesions had spontaneously disappeared or left calcific residues. It is concluded that in Indian epileptic patients with SSECTL cysticercosis is the commonest aetiology. A treatment protocol for these patients is suggested on the basis of the findings.
\end{abstract}

Department of Neurological Sciences and Pathology, $\star$ Christian Medical College Hospital, Vellore, India $M \mathrm{~J}$ Chandy V Rajshekhar $S$ Ghosh

$S$ Prakash

T Joseph

J Abraham

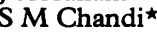

Correspondence to Dr Rajshekhar Department of Neurosurgery, Dartmouth Hitchcock Medical Cente Bldg 50, Hanover, New Hampshire 03756, USA Presented in part at the 38th Annual Conference of the Chandigarh, 18 to 20 December 1988.

Received 10 April 1989 and in final revised form 19 November 1990. Accepted 6 December 1990
Since the advent of CT scan in India a large number of patients with focal or generalised seizures are being investigated for structural cerebral lesions. A significant number (26\%) of these patients are found to have single, small ( $<10 \mathrm{~mm}$ ) enhancing CT lesion (SSECTL) with varying amount of surrounding oedema. ${ }^{1}$ The exact pathological nature of these lesions has not been verified as biopsy of such lesions has only been carried out in isolated cases. Various presumptive diagnoses such as tuberculoma, cysticercosis, sarcoidosis, larva migrans, transient viral encephalitis, microabscesses, post ictal enhancement and vascular lesions have been considered. ${ }^{1-6}$ Due to the high incidence of tuberculosis in our population and the "response" of the lesions to anti-tuberculous drugs, it was widely believed that these lesions were tuberculomas. Sethi et al ${ }^{7}$ reported that these lesions showed a spontaneous resolution and they were popularly termed "disappearing" lesions. This led to further confusion and controversy on the aetiology of these lesions.

The aims of our study were twofold; firstly to determine the pathological nature of SSECTL in a large series of consecutive patients; and secondly to rationalise the management of patients with such lesions.

\section{Material and methods}

From May 1987 till October 1988, 30 consecutive patients were included in this study with the following criteria: A) history of focal or generalised seizures; B) presence of a single, small ( $<10 \mathrm{~mm}$ ), enhancing lesion on the CT scan (SSECTL); C) no history of anti-tuberculous or other specific drug therapy for the lesions.

The patients' ages ranged from seven to 53 years, most of them being in the second to fourth decades. There were an equal number of males and females (15 each).

All except one patient presented with focal seizures with or without secondary generalisation. One patient had generalised seizures. The duration of symptoms at presentation ranged from 10 days to five years. Eleven patients complained of headaches. Neurological examination revealed minimal deficit in 23 patients in the form of soft signs. One patient had moderate distal hemiparesis (grade 4/5). Seven patients had no neurological deficits. None of these patients had evidence of raised intracranial tension.

The majority of these lesions were situated in and around the sensorimotor area (frontoparietal) (23/30). Five lesions were situated in the posterior parietal and two in the temporal regions. All lesions were situated superficially in the grey matter or at the grey-white junction. Most of them were found in supraventricular cuts $(25 / 30)$. All lesions were isodense in the plain CT scan with significant enhancement on the contrast scans. There were 17 "ring" lesions (central hypodensity with peripheral enhancement) and 13 "disc" lesions (lesion enhancing uniformly). There was a variable amount of oedema around the lesions. A midline shift was not seen in any case but mass effect was present in the form of effacement of the surrounding sulci.

The patients were grouped into three categories on the basis of their management: group A: patients who did not have a biopsy 

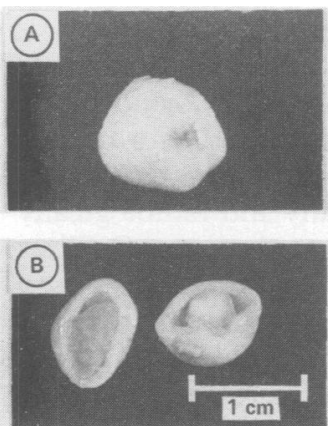

Figure 1 A) a nodule excised from a patient in group C. B) cut section of the same with a mural nodule. (five patients); group B: patients who had a CT guided stereotaxic biopsy (CTGSB) (10 patients); and group C: patients who had an excision biopsy of their lesions following CT guided stereotaxic localisation (CTGSE) (15 patients).

CT guided stereotaxic biopsy was originally planned for all these patients. However, prebiopsy CT scans showed a disappearance of the lesions in three patients. Two other patients who were being followed up with CT scans for their lesions over the last three years and attended the department during the period of the study were also included. These five patients constitute group A. CTGSB was carried out on the next ten patients (group B). The material obtained from the stereotaxic biopsy was insufficient to arrive at a definitive diagnosis and thus in the last 15 patients (group C) the lesions were excised.

The CT guided stereotaxic procedures were performed using the Brown-Roberts-Wells (BRW) system (Radionics, Burlington, Mass, USA). We found the Azimuth-Declination method ideal for biopsy and localisation. ${ }^{8}$ The lesions were excised through a trephine craniotomy after localisation. In the first four patients of group C, the excised nodules were bisected in the operating room and then put into formalin. The next nine nodules were sent intact. In another two patients a well-defined nodule was not found.

Twenty-six patients were treated with anticonvulsants (phenytoin sodium, phenobarbitone or carbamazepine). This includes all patients in groups A and B and 11 patients in group $C$. The remaining four patients from group $\mathrm{C}$ were not given anticonvulsants following excision of their lesions but they did receive them preoperatively.

All except three patients in groups A and B had follow up scans usually at three months after initial presentation. In the case of patients in group $\mathrm{C}$ whose lesions were totally excised, follow up scans were not carried out. Two patients of group $\mathrm{C}$ who did not have a total excision of the lesion had follow up scans.

\section{Results}

Group $B$ Diagnosis in this group of patients was based on the smears of the biopsy specimen. All specimens showed gliosis with or without chronic inflammation characterised mainly by lymphocytes and occasional plasma

Figure 2 Biopsy from a patient in group $C$ showing a cysticercus with the sucker and part of the body $(H \in E \times 160)$
Table 1 Histopathological diagnosis in patients in groups $B$ and $C$

\begin{tabular}{ll}
\hline Diagnosis & $\begin{array}{l}\text { Number of } \\
\text { patients }\end{array}$ \\
\hline Cysticercus with granulomatous inflammation & 7 \\
Cavitary lesion suggestive of parasitic granuloma & 5 \\
Hyalinised nodule with chronic inflammation & 1 \\
Gliosis with or without inflammation & $12^{\star}$ \\
\hline
\end{tabular}

*Includes all 10 patients of group B and 2 of group C.

cells. In two patients there was a suggestion of a granuloma with epithelioid cells.

Group $C$ In 13 cases, a well-defined, firm, white nodule was excised (figs $1 \mathrm{~A}$ and $\mathrm{B}$ ). The diameter of the nodules varied between 0.8 and $1 \cdot 2 \mathrm{~cm}$. Central cavitation was seen on sectioning 12 of them.

Microscopic examination showed a cyst containing parts of an intact or degenerated cysticercus in seven cases (fig 2 ). The cysts were surrounded by chronic inflammatory cells. Cavitary lesions with convoluted margins were seen in five other cases. The cavities contained necrotic material and were lined by palisaded epithelioid histiocytes and surrounded by dense collections of lymphocytes, plasma cells, eosinophils, epithelioid cells and foreign body granulomata. The surrounding brain tissue showed gliosis and a variable amount of fibrosis in all 12 cases. In one patient a well-defined, solid, collagenised nodule was seen. There was no specific lesion seen in this specimen. In two others microscopic examination only revealed gliosis and chronic inflammation. Caseous necrosis was not seen in any case. Table 1 summarises the histopathological diagnoses in groups $\mathrm{B}$ and $\mathrm{C}$. The pathological findings in group $\mathrm{C}$ have been reported earlier. ${ }^{9}$

Table 2 summarises the findings on follow up CT scans in patients of groups A and B.

Group $A$ In three patients the lesions had either "disappeared" or had been reduced to a calcific dot, within four to six weeks of the initial scan. In two others the lesion decreased in size on CT scans carried out after one, and one and a half years after initial presentation. In scans carried out at three years after the first ones, the lesion had "disappeared" in one and left a calcific dot in another.

Group B The lesions "disappeared" in three, became smaller in one and were reduced to calcific dots in three others. Three patients were lost to follow up.

Group C Two patients who did not have total excision of their lesions had follow up scans. In one the repeat scan after three months showed no change, but that taken at nine months showed a "disappearance" of the lesion. In the other patient the lesion became ill-defined and slightly larger after three months. This patient

Table 2 CT findings at last follow up (groups $A$ and B)

\begin{tabular}{ll}
\hline CT findings & $\begin{array}{l}\text { Number of } \\
\text { patients }\end{array}$ \\
\hline "Disappeared" & 6 \\
Calcific dot & 5 \\
Reduced size & 1 \\
Same or larger size & 0 \\
No follow up & 3 \\
\hline
\end{tabular}


who refused further surgery is being followed on anticonvulsant therapy.

Four patients had procedure related complications. One patient had a moderate sized cerebral haematoma following CTGSB. One patient had increased facial weakness after an attempt at excision of a lesion. A third patient developed transient motor dysphasia and the last one had marked hemiparesis after excision of the lesion. The post operative CT scan in the latter patient showed a marked increase in cerebral oedema around the operative site. All these patients were given steroids for a week to ten days, with which they all made a significant recovery.

\section{Discussion}

Technical difficulties in the biopsy of SSECTL The pathological nature of SSECTL has never been verified in a large series of consecutive patients. The major limiting factors have been the small size of these lesions which makes operative localisation difficult if not impossible, and the location of the lesions in eloquent areas, which increases the risk of producing post operative deficits. CT guided stereotaxic surgery enabled us to localise the lesions precisely and avoid these problems in the majority of our patients. Based on the findings in the first six patients of group B, we concluded that these lesions were "focal encephalitides" of unknown aetiology. ${ }^{10}$ It was obvious that more tissue was needed to arrive at an aetiological diagnosis and thus we decided on excision biopsy of the lesions.

The first four nodules, sent after sectioning in the operating room, showed microscopic evidence of a parasitic granuloma but did not reveal the causative agent. The subsequent nine specimens were sent intact and in seven cysticerci were identified. It is possible that the inconclusive nature of the biopsy in the first four cases was due to the loss of the cysticercus following bisection in the operating room.

These lesions are generally very firm or hard in consistency. This is the likely reason for the inconclusive biopsies in group $B$. The stereotaxic biopsy instruments are blunt tipped and they will not penetrate the thick capsules of these lesions. It is possible that the lesions were pushed away and the tissue that was obtained was from the surrounding brain. A similar problem is encountered with other inflammatory lesions as well. ${ }^{11}$

Figure 3 Suggested management protocol for patients with SSECTL. SSECTL = single, small, enhancing CT lesion, $C T S G E=C T$ guided stereotaxic localisation and excision.
"Disappearing" CT lesions and neurocysticercosis

In 12 out of 15 patients in group $A$ and $B$, the lesions "disappeared" or turned into calcific dots with no specific therapy. The patients in these groups were clinically and radiologically indistinguishable from those in group C. Thus these 30 patients form a homogeneous group and the pathological findings in group $C$ represent the pathology of "disappearing" CT lesions. We are therefore convinced that "disappearing" lesions are nothing but a manifestation of a very benign form of neurocysticercosis.

In retrospect, cysticercosis should have been the obvious choice as the aetiology of these lesions. Cerebral cysticercosis, the commonest parasitic infestation of the brain is frequently "silent" and even in those in whom it produces symptoms, it can be a benign disorder. ${ }^{12-14}$ Eighty per cent of patients with benign cerebral cysticercosis present with seizures. ${ }^{13}$ Besides this, spontaneous radiographic resolution of cerebral cysticercosis was reported by Miller $e t$ $a l^{15}$ in 1983. "Disappearance" or death and calcification of these parasites in the brain is a natural event in the evolution of most types of benign cerebral cysticercosis. This process usually takes from 18 months to 10 years from the time of infestation. ${ }^{14}{ }^{15}$ The rapid resolution of the granuloma ("disappearance" in four to six weeks) in some of our patients and the long duration of symptoms in others is difficult to explain except by postulating that this is determined by the individual host-parasite reaction. Because of this variability in the natural history of these lesions it is difficult to set an absolute time limit beyond which to biopsy a stable but persistent lesion.

Serological tests in the diagnosis of SSECTL Immunological tests have been used in the diagnosis of neurocysticercosis. The specificity and sensitivity of the tests are lower in patients with the benign form of the disease. ${ }^{16}$ ELISA has been used to detect anti-cysticercus antibodies in the serum or CSF of patients with SSECTL. Ahuja et al reported that only a third of their patients with SSECTL had a positive result with this assay. In a collaborative study with the Centers for Disease Control, Atlanta, USA, we studied the serum of 18 patients with SSECTL, using the immunoblot assay for cysticercus antibodies. ${ }^{18}$ This assay reportedly has a higher specificity and sensitivity than the earlier tests. ${ }^{19}$ However, this test was positive in only one patient. Thus we feel that the immunological tests are useful only if they are positive and a negative test does not exclude the diagnosis of neurocysticercosis. Serological tests could, however, be used as an initial noninvasive test in the diagnosis of these lesions.

\section{Management protocol for patients with} SSECTL

Since none of our biopsies showed evidence of a tuberculous lesion, empirical anti tuberculous therapy, as a routine treatment for these lesions should be avoided. We propose a rational 
management for patients with SSECTL on the basis of the findings in our study (fig 3). Conservative treatment with anticonvulsants and monitoring the lesion with CT forms the mainstay of our management protocol. Stereotaxic biopsy or excision biopsy is neither recommended nor required for the majority of patients with SSECTL. Serological tests, if positive, may help in avoiding a biopsy for stable but persistent lesions. A pathological diagnosis is only required if the lesions increase in size or do not resolve spontaneously within a reasonable period of time. CT guided stereotaxic localisation and excision of the lesion gives the best chance of obtaining a definitive diagnosis.

Indian patients with seizures and single, small, enhancing CT lesions most often have a benign, self-limiting disorder. The lesions described in our study are the same as "disappearing" CT lesions and most of them represent cerebral cysticercosis in various stages of the natural evolution of the disease. Most of these patients can be treated conservatively with anticonvulsants. Surgical excision for pathological verification is only required for enlarging and possibly for persistent lesions.

1 Wadia RS, Makhale CN, Kelkar AV, Grant KB. Focal epilepsy in India with special reference to lesions showing ring or disc-like enhancement on contrast computed tomography. J Neurol Neurosurg Psychiatry 1987;50: 1298-301.

2 Bhargava S, Tandon PN. CNS tuberculosis. Lessons learnt from CT studies. Neurol India 1980;28:207-12.

3 Bhatia S, Tandon PN. Solitary "microlesions" in CT-a clinical study and follow up. Neurol India 1988;36:139-50.

4 Vengsarkar US, Pisipaty RP, Parekh B, Panchal VG, Shetty MN. Intracranial tuberculoma and the CT scan. J Neurosurg 1986;64:568-74.

5 Beyl DZD, Hermanus N, Colle H, Goldman S. Focal seizures with reversible hypodensity on the CT scan. seizures with reversible hypodensity on the
J Neurol Neurosurg Psychiatry 1985;48:187-8.

6 Basauri L, Zuleta A, Loayza P, Olivares A. Microabscesses and presumptive inflammatory nodules of the brain. Acta Neurochir 1983;68:27-32.

7 Sethi PK, Kumar BR, Madan VS, Mohan V. Appearing and disappearing CT scan abnormalities and seizures. $J$ Neurol Neurosurg Psychiatry 1985;48:866-9.

8 Chandy MJ, Rajshekhar V. Computed tomography guided stereotactic surgery. In: Sinha KK, ed. Progress in Clinical Neurosciences. Ranchi: Neurological Society of India 1988;2:19-28.

9 Chandy MJ, Rajshekhar V, Prakash S, et al. Cysticercosis causing single small CT lesions in Indian patients with seizures (Letter). Lancet 1989;i:390-1.

10 Chandy MJ, Rajshekhar V. Focal epilepsy in India (letter). J Neurol Neurosurg Psychiatry 1988;51:1242.

11 Rajshekhar V, Abraham J, Chandy MJ. Avoiding empiric therapy for brain masses in Indian patients using CT therapy for brain masses in Indian patients using

12 Showramma A, Reddy DB. Silent cysticercosis of the brain. An analysis of five cases with special reference to histopathology. Indian J Pathol Bacteriol 1983;6:142-7.

13 Estanol B, Corona T, Abad P. A prognostic classification of cerebral cysticercosis: therapeutic implications. J Neurol Neurosurg Psychiatry 1986;49:1131-4.

14 Grisolia JS, Wiederholt WC. CNS cysticercosis. Arch Neurol 1982;39:540-4.

15 Miller B, Gardner B, Goldberg M, Heiner D. Spontaneous radiographic disappearance of cerebral cysticercosis. Three cases. Neurology 1983;33:1377-9.

16 Rosas N, Sotelo J, Nieto D. ELISA in the diagnosis of neurocysticercosis. Arch Neurol 1986;43:353-6.

17 Ahuja GK, Behari M, Prasad K, Goulatia RK, Jailkhani BL. Disappearing CT lesions in epilepsy: is tuberculosis or
cysticercosis the cause? J Neurol Neurosurg Psychiatry cysticercosis the

18 Rajshekhar V, Wilson M, Schantz PM. Cysticercus immunoblot assay in Indian patients with single small CT lesions (Letter). J Neurol Neurosurg Psychiatry (in press)

19 Tsang VCW, Brand JA, Boyer AE. An enzyme linked immunotransfer blot assay and glycoprotein antigens for diagnosing human cysticercosis (Taenia solium). Journal of Infectious Diseases 1989;159:50-9. 